\title{
GAUSSIAN RANDOM SETS IN BANACH SPACE*
}

\author{
M. L. PURI, ${ }^{+}$D. A. RALESCU† AND S. S. RALESCU
}

\begin{abstract}
We define a Gaussian random set in a Banach space, and we prove the following characterization theorem: Every Gaussian random 'set can be represented as the sum of its expected value and a Gaussian mean zero random element.

1. Introduction. The concept of random set (Robbins [16], [17]), Matheron [11]) has received considerable attention in recent years, partly motivated by geometric probability considerations and partly as an application of probability techniques in Banach space. The strong law of large numbers for random sets in $\mathbf{R}^{p}$ was proved by Artstein and Vitale [1] and for random sets in Banach space by Puri and Ralescu [12], [13]. The central limit theorem (CLT)
\end{abstract}

* Received by the editors November 8, 1985.

$\dagger$ Research supported by a National Science Foundation Grant. 
for random sets in $\mathbf{R}^{p}$ was proved in a particular case by Cressie [5] and in a general set-up by Weil [18]. In a Banach space setting, the CLT was given by Giné, Hahn and Zinn [8] and a more general result by Puri and Ralescu [13].

In view of statistical applications, the concept of normality for random sets should play an important role. Such a concept was defined by Lyashenko [10] in $\mathbf{R}^{p}$, who also gave a characterization of normal random sets.

In this paper, we introduce the concept of a Gaussian random set in a separable Banach space $\mathscr{X}$ and characterize such a set as the sum of a constant set and a Gaussian random element (of $\mathscr{X}$ ).

In $\S 2$ we give some preliminaries on random sets and their expectations. Section 3 deals with an embedding of random compact sets into random elements of the space of continuous functions on some compact set. Finally, in $\S 4$ we define Gaussian random sets in $\mathscr{Z}$ and prove the characterization theorem.

2. Random sets. Let $\mathscr{X}$ denote a separable Banach space with norm $\|\cdot\|, \mathscr{X}^{*}$ the dual of $\mathscr{X}$, and $\langle\cdots\rangle: \mathscr{L}^{*} \times \mathscr{X} \rightarrow \mathbf{R}$ the duality map: $\left\langle x^{*}, x\right\rangle=x^{*}(x)$ for $x^{*} \in \mathscr{X}^{*}, x \in \mathscr{X}$.

If $(\Omega, \mathscr{A}, \mathbf{P})$ is a probability space, a random element in $\mathscr{X}$ is a Borel measurable function $f: \Omega \rightarrow \mathscr{X}$. The expected value $\mathbf{E} f$ is defined in terms of the Bochner integral; it exists if $\mathbf{E}\|f\|<\infty$.

A random element $f$ is Gaussian if $\left\langle x^{*}, f\right\rangle$ is a normal random variable for any $x^{*} \in \mathscr{X}^{*}$.

We will be interested in random elements in $C(M)$, the Banach space of continuous functions on the compact, Hausdorff space $M$. It follows easily from the Riesz representation theorem that if $f: \Omega \rightarrow C(M)$ is Gaussian, then the vector $\left(f\left(t_{1}\right), \cdots, f\left(t_{n}\right)\right)$ is (multivariate) normal, for any $t_{1}, \cdots, t_{n} \in M$. Let $\mathscr{K}(\mathscr{X})$ denote all the nonempty compact subsets of $\mathscr{X}$, and let $\mathscr{K}_{c}(\mathscr{X})$ denote the nonempty compact convex subsets of $\mathscr{X}$. The space $\mathscr{K}(\mathscr{X})$ is a metric space with respect to the Hausdorff metric:

$$
d(A, B)=\max \left\{\sup _{a \in A} \inf _{b \in B}\|a-b\|, \sup _{b \in B} \inf _{a \in A}\|a-b\|\right\} \text { for } A, B \in \mathscr{K}(\mathscr{X}) .
$$

More precisely, $(\mathscr{K}(\mathscr{X}), d)$ is a complete, separable metric space, and $\mathscr{K}_{c}(\mathscr{X})$ is a closed subspace (see Debreu [6]).

The space $\mathscr{K}(\mathscr{X})$ has a linear structure induced by the operations:

$$
A+B=\{a+b \mid a \in A, b \in B\}, \lambda A=\{\lambda a \mid a \in A\} \text { for } A, B \in \mathscr{K}(\mathscr{X}), \lambda \in \mathbf{R} .
$$

Note however that $\mathscr{K}(\mathscr{X})$ is not a vector space (it is not a group with respect to addition).

Denote $\|A\|=d(A,\{0\})$ for $A \in \mathscr{K}(\mathscr{X})$.

As before, let $(\Omega, A, \mathbf{P})$ be a probability space. A random set (in $\mathscr{X})$ is a Borel measurable function $X: \Omega \rightarrow \mathscr{K}(\mathscr{X})$. The expected value $\mathbf{E} X$ is defined as $\mathbf{E} X=\left\{\mathbf{E} f \mid f \in L^{1}(\Omega, A, \mathbf{P}), f(\omega) \in\right.$ $\mathbf{X}(\omega)$ a.e. where $f: \Omega \rightarrow \mathscr{X}$ is a selection of $X$ (Aumann [2]). If $\mathbf{E}\|\boldsymbol{X}\|<\infty$, then $\mathbf{E} X \in \mathscr{K}(\mathscr{X})$. Another definition of the expected value is due to Debreu [6]: first, the space $\mathscr{K}_{c}(\mathscr{X})$ is embedded into a Banach space (via the Rådström embedding theorem [14]) and then one proceeds as in the case of the Bochner integral. If $\mathbf{E}\|X\|<\infty$, the two definitions of $\mathbf{E} X$ are equivalent (Byrne [3]).

In what follows we consider random sets with values in $\mathscr{K}_{c}(\mathscr{X})$, i.e., random convex sets.

3. The embedding theorem. An isometric embedding of $\mathscr{K}_{c}(\mathscr{X})$ into a separable Banach space of functions can be obtained from a theorem of Hörmander [9] (see also Giné, Hahn and Zinn [8]).

Let ball $\mathscr{X}^{*}=\left\{x^{*} \in \mathscr{X}^{*} \mid\left\|x^{*}\right\| \leqq 1\right\}$ denote the unit ball of $\mathscr{Q}^{*}$, let $w^{*}$ denote the weak topology in $\mathscr{X}^{*}$, and $C$ (ball $\left.\mathscr{X}^{*}, w^{*}\right)$, the Banach space of functions defined on ball $\mathscr{X}^{*}$ and continuous in weak* topology.

THEOREM 3.1. There exists a function $j: \mathscr{K}_{c}(\mathscr{X}) \rightarrow C\left(\right.$ ball $\left.\mathscr{X}^{*}, w^{*}\right)$ such that

(a) $j$ is an isometry (i.e., $\|j(K)-j(L)\|_{\infty}=d(K, L)$ ).

(b) $j(K+L)=j(K)+j(L)$.

(c) $j(\lambda K)=\lambda j(K), \lambda \geqq 0$.

Proof. From Hörmander [9, Theorem 8], it follows that there is an isometry $j$ satisfying (b) and $(\mathrm{c})$, from $\mathscr{K}_{c}(\mathscr{X})$ into the space of bounded continuous functions on ball $\mathscr{X}^{*}$. Since each $K \in \mathscr{K}_{c}(\mathscr{X})$ is totally bounded, it follows that $j(K) \in C$ (ball $\left.\mathscr{X}^{*}, w^{*}\right)$.

Note. Since (ball $\left.\mathscr{L}^{*}, w^{*}\right)$ is compact and metrizable, the space $C\left(\right.$ ball $\left.\mathscr{X}^{*}, w^{*}\right)$ is separable. The isometry $j$ is defined via the support function of a compact set: $j(K)=s_{K}$ where $s_{K}\left(x^{*}\right)=$ $\sup _{a \in K}\left\langle x^{*}, a\right\rangle, x^{*} \in$ ball $\mathscr{Q}^{*}$. 
Actually, $s_{K}$ can be defined on $\mathscr{X}^{*}$ as above, and has the following simple properties:

(i) $s_{K}$ is subadditive, i.e., $s_{K}\left(x^{*}+y^{*}\right) \leqq s_{K}\left(x^{*}\right)+s_{K}\left(y^{*}\right)$ for $x^{*}, y^{*} \in X^{*}$.

(ii) $s_{K}$ is positively homogeneous, i.e., $s_{K}\left(\lambda x^{*}\right)=\lambda s_{K}\left(x^{*}\right)$ for $x^{*} \in X^{*}, \lambda \geqq 0$.

In view of this embedding theorem, any random set $X: \Omega \rightarrow \mathscr{K}_{c}(\mathscr{X})$ can be thought of as a random element of $C$ (ball $X^{*}, w^{*}$ ) by considering $j(X)=s_{X}: \Omega \rightarrow C$ (ball $\mathscr{X}^{*}, w^{*}$ ), where $s_{X}(\omega)=s_{X(\omega)}$.

The following result will be useful. ball $X^{*}$

LEMMA 3.1. If $X: \Omega \rightarrow \mathscr{K}_{c}(\mathscr{X})$ satisfies $\mathbf{E}\|X\|<\infty$, then $\mathbf{E}\left[s_{X}\left(x^{*}\right)\right]=s_{\mathbf{E} X}\left(x^{*}\right)$ for $x^{*} \in$

Proof. It is easily seen that the result holds if $X=\sum_{i=1}^{n} x_{A_{i}} K_{i}$ where $A_{i} \in \mathscr{A}, K_{i} \in \mathscr{K}_{c}(\mathscr{X})$ and $x_{A_{i}}$ denotes the characteristic function of $A_{i}$ (i.e., if $X$ is a simple function). The proof follows by using the definition of the Debreu expected value, the fact that $j$ is an isometry, and the Lebesgue dominated convergence theorem.

4. Gaussian random sets. The concept of a normal random set in $\mathbf{R}^{p}$ was defined by Lyashenko [10] who also characterized such random sets. His proof of characterization, however, is strongly dependent on the finite dimensionality assumption. We define here a Gaussian random set in an arbitrary separable Banach space, and we represent such a random set as the sum of a constant set and a Gaussian random element.

Definition 4.1. A random set $X: \Omega \rightarrow \mathscr{K}_{c}(\mathscr{X})$ is Gaussian if $s_{X}$ is a Gaussian random element of $C$ (ball $\left.\mathscr{X}^{*}, w^{*}\right)$.

This definition implies (see $\S 2)$ that $\left(s_{X}\left(x_{1}^{*}\right), \cdots, s_{X}\left(x_{n}^{*}\right)\right.$ is normal for every $x_{1}^{*}, \cdots, x_{n}^{*} \in$ ball $\mathscr{L}^{*}$. Consequently $\left(s_{X}\left(x_{1}^{*}\right), \cdots, s_{X}\left(x_{n}^{*}\right)\right)$ is normal for every $x_{1}^{*}, \cdots, x_{n}^{*} \in \mathscr{X}^{*}$ (since $s_{X}$ is positively homogeneous; see property (ii) in $\$ 3$ ).

It is obvious that if $X$ and $Y$ are independent Gaussian random sets, then $X+Y$ is Gaussian. Also $\lambda X$ is Gaussian $(\lambda \geqq 0)$ whenever $X$ is Gaussian.

The following theorem characterizes the Gaussian random sets in $\mathscr{X}$.

THEOREM 4.1. Let $X: \Omega \rightarrow \mathscr{K}_{c}(\mathscr{X})$ be a random set with $\mathbf{E}\|X\|<\infty$. The following statements are equivalent.

(i) $X$ is Gaussian.

(ii) $X=\mathbf{E} X+\{\xi\}$ where $\xi$ is a Gaussian random element in $X$ with mean zero.

Proof. (ii) $\Rightarrow$ (i). From $X=\mathbf{E} X+\{\xi\}$, it follows that $s_{X}=s_{\mathbf{E X}}+\langle\cdot, \xi\rangle$. Thus, if $\varphi^{*} \in$ $\left(C\left(\text { ball } \mathscr{X}^{*}, w^{*}\right)\right)^{*}$, then $\varphi^{*}\left(s_{X}\right)=\varphi^{*}\left(s_{\mathbf{E} X}\right)+\varphi^{*}(\langle\cdot, \xi\rangle)$.

Denote $\Phi(x)=\varphi^{*}(\langle\cdot, x\rangle)$. Clearly $\Phi$ is linear, and since $|\Phi(x)| \leqq\left\|\varphi^{*}\right\|\|x\|$, it follows that $\Phi \in \mathscr{L}^{*}$. Thus $\langle\Phi, \xi\rangle=\varphi^{*}(\langle\cdot, \xi\rangle)$ is a normal random variable which implies that $\varphi^{*}\left(s_{X}\right)$ is normal. Consequently, $X$ is Gaussian.

We now prove that (i) $\Rightarrow\left(\right.$ ii). Let $s_{X}$ be Gaussian. Define $\psi: \Omega \rightarrow C$ (ball $\mathscr{L}^{*}, w^{*}$ ) by $\psi=s_{X}-s_{\mathrm{EX}}$. Fix an $\omega \in \Omega$. Then $\psi(w)=s_{X(\omega)}-s_{\mathbf{E X}}$.

CLAIM 1. $\psi(\omega)$ is linear and weak*-continuous on $\mathscr{2}^{*}$.

Let $\lambda_{1}, \cdots, \lambda_{n} \geqq 0$ and $x_{1}^{*}, \cdots, x_{n}^{*} \in \mathscr{Q}^{*}$ be fixed. Then $\sum_{i=l}^{n} \lambda_{i} s_{X}\left(x_{i}^{*}\right)-s_{X}\left(\sum_{i=l}^{n} \lambda_{i} x_{i}^{*}\right) \geqq 0$ (from properties (i) and (ii) in $\S 3$ ). By hypothesis, $\left(s_{X}\left(x_{1}^{*}\right), \cdots, s_{X}\left(x_{n}^{*}\right), s_{X}\left(\sum_{i=1}^{n} \lambda_{i} x_{i}^{*}\right)\right.$ ) is normal which implies that $\sum_{i=l}^{n} \lambda_{i} s_{X}\left(x_{i}^{*}\right)-s_{X}\left(\sum_{i=l}^{n} \lambda_{i} x_{i}^{*}\right)$ is a normal random variable. From the above inequality, it follows that this random variable must be degenerate, i.e.,

$$
\begin{aligned}
\sum_{i=1}^{n} \lambda_{i} s_{X}\left(x_{i}^{*}\right)-s_{X}\left(\sum_{i=1}^{n} \lambda_{i} x_{i}^{*}\right) & =\mathbf{E}\left[\sum_{i=1}^{n} \lambda_{i} s_{X}\left(x_{i}^{*}\right)-s_{X}\left(\sum_{i=1}^{n} \lambda_{i} x_{i}^{*}\right)\right] \\
& =\sum_{i=1}^{n} \lambda_{i} s_{\mathbf{E} X}\left(x_{i}^{*}\right)-s_{\mathbf{E X}}\left(\sum_{i=1}^{n} \lambda_{i} x_{i}^{*}\right),
\end{aligned}
$$

the last equality following from Lemma 3.1.

Thus $\sum_{i=1}^{n} \lambda_{i} \psi(\omega)\left(x_{i}^{*}\right)=\psi(\omega) \sum_{i=1}^{n}\left(\lambda_{i} x_{i}^{*}\right)$. Take now $\lambda_{i}=1, \quad i=1, \cdots, n$. We get $\psi(\omega)\left(\sum_{i=1}^{n} x_{i}^{*}\right)=\sum_{i=1}^{n} \psi(\omega)\left(x_{i}^{*}\right)$, implying that $\psi(\omega)$ is additive. Take $x_{1}^{*}=x^{*}, x_{2}^{*}=-x^{*}, \lambda_{1}=$ $\lambda_{2}=1$. We get $\psi(\omega)\left(-x^{*}\right)=-\psi(\omega)\left(x^{*}\right)$, implying that $\psi(\omega)$ is linear.

Since $\psi(\omega)$ is weak*-continuous on ball $\mathscr{X}^{*}$, it follows from Lemma 5.1 (in the Appendix) that $\psi(\omega)$ is weak ${ }^{*}$-continuous on $\mathscr{X}^{*}$. It is well known that such a $\psi(\omega)$ is representable as $\psi(\omega)\left(x^{*}\right)=\left\langle x^{*}, \xi(\omega)\right\rangle, x^{*} \in \mathscr{X}^{*}$ for some $\xi(\omega) \in \mathscr{X}$ (see, for example, Yoshida [19, p. 112]).

Claim 2. $\xi: \Omega \rightarrow \mathscr{X}$ is measurable.

Indeed, since $\left\langle x^{*}, \xi\right\rangle=\psi(\cdot)\left(x^{*}\right)=s_{X}\left(x^{*}\right)-s_{\mathbf{E} X}\left(\tau^{*}\right)$, it follows that $\left\langle x^{*}, \xi\right\rangle$ is measurable for each $x^{*} \in \mathscr{X}^{*}$. Using the Pettis theorem (Yoshida [19, p. 131]), it follows that $\xi$ is strongly measurable (since $\mathscr{X}$ is separable). 
Claim 3. $\xi$ is mean zero Gaussian.

Since $\left\langle x^{*}, \xi\right\rangle=s_{X}\left(x^{*}\right)-s_{\mathbf{E X}}\left(x^{*}\right)$, it follows that $\left\langle x^{*}, \xi\right\rangle$ is a normal mean zero random variable, for each $x^{*} \in \mathscr{Q}^{*}$.

To conclude the proof of the theorem, we write $s_{X}\left(x^{*}\right)=s_{\mathbf{E X}}\left(x^{*}\right)+\left\langle x^{*}, \xi\right\rangle=$ $s_{\mathbf{E} X}\left(x^{*}\right)+s_{\{\xi\}}\left(x^{*}\right)=s_{\mathbf{E X}+\{\xi\}}\left(x^{*}\right)$, implying that $X=\mathbf{E} X+\{\xi\}$, giving the desired representation.

5. Appendix. The following lemma is used in the proof of Theorem 4.1.

LEMMA 5.1. Let $\varphi: \mathscr{X}^{*} \rightarrow \mathbf{R}$ be linear and weak*-continuous on ball $\mathscr{L}^{*}$. Then $\varphi$ is weak*. continuous on $\mathscr{Q}^{*}$.

Proof. It suffices to show that $\{\varphi \leqq c\}$ is weak*-closed for any $c \in \mathbf{R}$. Obviously $\{\varphi \leqq c\}$ is convex, and so, by the Krein-Smulian theorem (see Dunford and Schwartz [7, p. 429]), it is enough to show that $\{\varphi \leqq c\} \cap r$ (ball $\mathscr{X}^{*}$ ) is weak*-closed for all $r>0$, where $r$ (ball $\mathscr{X}^{*}$ ) is the ball with radius $r$.

Now $\{\varphi \leqq c\} \cap r$ (ball $\left.\mathscr{L}^{*}\right)=r\left[\{\varphi \leqq c / r\} \cap\right.$ ball $\left.\mathscr{L}^{*}\right]$ and $\{\varphi \leqq c / r\} \cap$ ball $\mathscr{X}^{*}$ is weak*-closed since $\varphi$ is weak*-continuous on ball $\mathscr{X}^{*}$. The proof follows.

\section{REFERENCES}

[1] Z. ARTSTein ANd R. A. Vitale, A strong law of large numbers for random compact sets, Ann. Probab., 3 (1975), pp. 879-882.

[2] R. J. AumanN, Integrals of set-valued functions, J. Math. Anal. Appl., 12 (1965), pp. 1-12.

[3] C. L. BYRne, Remarks on the set-valued integrals of Debreu and Aumann, J. Math. Anal. Appl., 62 (1978), pp. 243-246.

[4] N. CRessie, A strong limit theorem for random sets, Suppl. Adv. Appl. Probab., 10 (1978), pp. 36-46.

[5] — A central limit theorem for random sets, Z. Wahrsch. verw. Geb., 49 (1979), pp. 37-47.

[6] G. Debreu, Integration of correspondences, Proc. Fifth Berkeley Symposium on Mathematical Statistics and Probability. Vol. II. Univ. California Press, Berkeley, Los Angeles, 1966, pp. 351-372.

[7] N. Dunford AND J. SchwarTz, Linear Operators, Part 1: General Theory, Wiley Interscience, New York, 1958.

[8] E. Giné, M. C. HAhn ANd J. ZıNn, Limit Theorems for Random Sets: An Application of Probability in Banach Space Results, Lecture Notes in Mathematics 990, 1983, pp. 112-135.

[9] L. HÖRMANDER, Sur la fonction d'appui des ensembles convexes dans un espace localement convexe, Ark. Math., 3 (1954), pp. 181-186.

[10] N. N. LyAShENKO, The statistics of random compact sets of Euclidean space, Zap. Nauchn. Sem. Leningrad Otdel. Mat. Inst. Steklov, 98 (1980), pp. 115-139. (In Russian.)

[11] G. MAtheron, Random Sets and Integral Geometry, John Wiley, New York, 1975.

[12] M. L. PURi AND D. A. RAlesCu, Strong law of large number for Banach space-valued random sets, Ann. Probab., 11 (1983), pp. 222-224.

[13] - Limit theorems for random compact sets in Banach space, Math. Proc. Cambridge Phil. Soc., 97 (1985), pp. 151-158.

[14] H. RÅDSTRÖM, An embedding theorem for spaces of convex sets, Proc. Amer. Math. Soc., 3 (1952), pp. 165-169.

[15] B. D. RiPLEY, Foundations of random set theory, Ann. Probab., 4 (1976), pp. 995-998.

[16] H. E. Roвbins, On the measure of a random set, Ann. Math. Statist., 15 (1944), pp. 70-74.

[17] — On the measure of a random set. II, Ann. Math. Statist., 16 (1945), pp. 342-347.

[18] W. WEIL, An application of the central limit theorem for Banach space-valued random variables to the theory of random sets, Z. Wahrsch. verw. Geb., 60 (1982), pp. 203-208.

[19] K. YoshidA, Functional Analysis, Springer-Verlag, New York-Berlin-Heidelberg, 1965.

ON SEQUENCES OF RANDOM PROCESSES WITH TIGHT JUMP MAJORIZATION*

\section{A. LEBEDEV}

(Translated by Amal Ellis)

Let $Y$ be a complete separable metric space. Consider the set $D=D_{[0, \infty[}(Y)$ of càdlàg functions on $R_{+}=\left[0, \infty\left[\right.\right.$ with values in $Y$. Usually one considers the Skorokhod $J_{1}$-topology

\footnotetext{
${ }^{*}$ Received by the editors October 20, 1982; revised version May 30, 1983.
} 
Reproduced with permission of the copyright owner. Further reproduction prohibited without permission. 